\title{
Validity of the 32-item Hypomania Checklist (HCL-32) in a clinical sample with mood disorders in China
}

\author{
Hai-chen Yang ${ }^{1,2 \dagger}$, Cheng-mei Yuan ${ }^{3 \dagger}$, Tie-bang Liu ${ }^{2 \dagger}$, Ling-jiang Li $i^{*}$, Hong-jun Peng ${ }^{1}$, Chun-ping Liao ${ }^{2}$,
} Han Rong ${ }^{2}$, Yi-ru Fang ${ }^{3}$ and Jules Angst ${ }^{4}$

\begin{abstract}
Background: The 32-item Hypomania Checklist (HCL-32), a questionnaire for screening bipolar disorders, has been utilised in several countries, but it unclear if the Chinese version of the HCL-32 is valid.

Methods: Consecutive patients with bipolar disorders (BP, $N=300)$ and unipolar major depression (UP, $N=156)$ completed the Chinese version of the HCL-32. The subjects underwent a structured clinical interview for DSM-IV Axis-I disorders (SCID).

Results: The eigenvalues for the first three factors in the HCL-32 were calculated as 5.16 (active/elated), 2.72 (risktaking) and 2.48 (irritable) using factor analysis. Cronbach's alpha for the HCL-32 was calculated to be 0.88. Positive responses to twenty-eight items were significantly more frequent by patients with BP than those with UP, and the other four items (7th, 21st, 25th and 32nd) showed no such trend. Fourteen was the optimal cut-off for discriminating between BP and UP. The HCL-32 distinguished between BP-II and UP, with 13 being the optimal cut-off. A cut-off of 13 yielded a sensitivity of 0.77 and a specificity of 0.62 between BP and UP.

Conclusions: This study demonstrated that the simplified Chinese version of HCL-32 was valid for patients with mood disorders. The optimal cut-off of 13 for distinguishing between BP-II and UP was valid and could be used to improve the sensitivity of screening BP-II patients when the HCL-32 is used in psychiatric settings in China.
\end{abstract}

\section{Background}

It is important to differentiate bipolar disorders (BP) from other mood disorders; delayed diagnosis or misdiagnosis can prolong the suffering of patients [1-3] but accurate early diagnosis can be difficult [3,4]. As many as $40 \%$ of patients with bipolar disorders are initially misdiagnosed, and it can take as long as 10 years before these patients are diagnosed correctly [4]. In the general population, the misdiagnosis rate can be as high as $69 \%$ [5]. In China, $45.4 \%$ of outpatients with bipolar disorders are diagnosed incorrectly [6]. Bipolar patients often present in the depressive phase [7] and many patients with BP (particularly bipolar II) are diagnosed as having unipolar depressive disorder [3-8]. Clinical guidelines published by

\footnotetext{
* Correspondence: Ilj2920@163.com

+ Contributed equally

'Mental Health Institute, the 2nd Xiangya Hospital, Central South University,

No. 139 Renmin Zhong Road, Changsha 410011, China

Full list of author information is available at the end of the article
}

the American Psychiatric Association indicate that bipolar II disorder (BP-II) is often initially misdiagnosed as a major depressive disorder, leading to patients receiving incorrect treatments [9]. Hypomania, an element of bipolar II disorder, is not usually perceived by patients to be pathological and is not reported to clinicians $[10,11]$. The retrospective detection of hypomania is crucial for a correct diagnosis of bipolar disorder, particularly for BP-II. An instrument to detect hypomania retrospectively would be useful in clinical settings.

Recent studies have demonstrated that the 32-item Hypomania Checklist (HCL-32) developed by Jules Angst is a good screening instrument for past hypomanic episodes [12-15]. The HCL-32 is a self-administered questionnaire that screens for a history of hypomanic symptoms using thirty-two yes/no items and takes into account the subject's current mental state. The HCL-32 was demonstrated to have good sensitivity $(0.80)$ and

\section{Biomed Central}

(c) 2011 Yang et al; licensee BioMed Central Ltd. This is an Open Access article distributed under the terms of the Creative Commons Attribution License (http://creativecommons.org/licenses/by/2.0), which permits unrestricted use, distribution, and reproduction in any medium, provided the original work is properly cited. 
specificity (0.51) at an optimal cut-off of 14, in a sample comprising predominantly outpatients with BP and UP in Europe [12]. The HCL-32 can distinguish between BP and UP at a cut-off of 14 (sensitivity 0.82 and specificity 0.67) in Taiwan [14]. However, little is known about the usefulness of HCL-32 for patients with mood disorders in China. In China, simplified Chinese characters are used, whereas in Taiwan complicated Chinese characters are used. Furthermore, in Taiwan different terms are used to express anxiety and emotion in patients. Therefore, the Taiwanese version of the HCL-32 is difficult to use in mainland China.

The aim of this study was to evaluate the feasibility of using a simplified Chinese version of the HCL-32, to examine its psychometric properties and accuracy as a screening tool for bipolar disorders. The results were compared with those from previous studies concerning the use of the HCL-32 in various countries.

\section{Methods}

\section{Subjects}

Subjects from the outpatient and inpatient departments at Shenzhen and Shanghai mental health centres were enrolled in the study from January 2006 to December 2008. The Shenzhen Mental Health Centre is the only psychiatric hospital in Shenzhen city. The study was approved by the ethics committees of the two psychiatric hospitals.

Patients who satisfied the inclusion and exclusion criteria were evaluated. The inclusion criteria comprised patients diagnosed with major depressive disorder (unipolar depressive disorder, UP), bipolar I disorder (BP-I) or bipolar II disorder (BP-II), aged between 18 and 60 years, educated for a minimum of five years, and who provided written informed consent. The exclusion criteria comprised patients diagnosed with an unstable or severe clinical status, those who could not cooperate with the study procedures, patients who had received electroconvulsive therapy (ECT) or modified electroconvulsive therapy (MECT) during the previous four weeks, individuals who were illiterate, suffering from mental retardation, dementia or intellectual impairment. Subjects did not have to have a certain clinical status as the aim was to elucidate the relationship between current state and HCL-32 scores.

\section{Measure}

Upon consent from the author of the original HCL-32 (Jules Angst), the English version of the HCL-32 was translated into a simplified Chinese version. Back translation was performed by a bilingual psychiatrist unaware of the original HCL-32. A preliminary translated version was administered to individuals without psychiatric illness and patients with mood disorders. The authors reviewed the results of this preliminary investigation before producing the final version.
The contents of the HCL-32 were explained to the subjects and it was completed before the Structured Clinical Interview for DSM-IV Axis-I Disorders (SCID) was carried out; interviewers were blind to the HCL-32 results. All interviewers were psychiatrists with a minimum of five years experience. The kappa coefficient for diagnosis of bipolar disorders was 0.83 .

There were contents concerning rating of current mental states (much worse than usual, worse than usual, a little worse than usual, neither better nor worse than usual, a little better than usual, better than usual, much better than usual) in the HCL-32 in addition to the 32 items [12]. Subjects were asked to select one certain state.

\section{Statistical Analyses}

Principal component analysis with varimax rotation was used to determine the construct validity of the HCL-32. Eigenvalues $>1$ were initially retained and clinical considerations decided the final number of factors. The internal consistency of the HCL-32 was determined using Cronbach's alpha. Spearman correlation analysis was performed on the current mental state and the total score. Current mental states and the mean total HCL-32 scores were compared between groups using the Kruskal-Wallis test. The frequency of each symptom item and the total HCL-32 score were compared between groups using a t-test. The receiver operating characteristic (ROC) curve was used to distinguish between groups and to ascertain the sensitivity and specificity at various cut-offs. ROC curves can be difficult to understand. Therefore, the change in sensitivity and specificity at various cut-offs are presented in figures, rather than the ROC curve. Positive predictive value was defined as the proportion of subjects screened as positive for BP using the HCL-32 and having DSM-IV BP. Negative predictive value was defined as the proportion of subjects screened as negative for BP using the HCL-32 who had DSM-IV UP. Probability values less than 0.05 were considered statistically significant. All statistical analyses were carried out using SPSS-15.0 for Windows (SPSS, Chicago, IL, USA).

\section{Results}

\section{Description of samples}

Four hundred and fifty six subjects (232 from Shenzhen and 224 from Shanghai), including 197 outpatients and 269 inpatients, were enrolled in the study (Table 1). The mean age of BP patients was significantly lower than that of UP patients $(t=5.24, P<0.01)$.

\section{Frequency of positive responses}

The frequency of positive responses to twenty-eight items in BP patients was significantly higher than in UP 
Table 1 Description of samples

\begin{tabular}{lllll}
\hline & UP & BP & BP-I & BP-II \\
\hline $\mathrm{N}$ & 156 & 300 & 224 & 76 \\
$\%$ Female & 64.10 & 47.33 & 50.45 & 38.16 \\
Age (mean \pm & $40.34 \pm$ & $33.76 \pm$ & $33.78 \pm$ & $33.15 \pm$ \\
SD) & 14.23 & 11.69 & 10.67 & 14.04 \\
Education in & $10.21 \pm$ & $11.61 \pm$ & $11.23 \pm$ & $12.48 \pm$ \\
years & 2.78 & 3.40 & 3.45 & 3.11 \\
Married, \% & 71.15 & 65.33 & 62.50 & 72.37 \\
\hline
\end{tabular}

patients, with the exception of four items (7th item, tend to drive faster; 21 st item, more easily distracted; 25 th item, more impatient/irritable; 32nd item, take more drugs; Figure 1).

\section{Current mental state and HCL-32 self-assessment}

Mean HCL-32 scores were statistically different between groups, defined according to the current mental state of BP and UP (Table 2).

A significant $(P=0.02)$ but low positive correlation $(r$ $=0.13$ ) was demonstrated between current mental state and the HCL-32 score in BP patients $(N=300)$ using Spearman correlation analysis. Similar results were obtained for UP patients $(r=0.23, P<0.01, N=156)$.

\section{Factor analysis}

Analysis of data concerning subjects with mood disorders $(N=456)$ using principal component analysis with varimax rotation, revealed that the eigenvalues of seven factors were greater than 1, and this explained 51.04\% of the total variance. The eigenvalues of factors I, II, and III were 5.16, 2.72, and 2.48, respectively (other factors had eigenvalues $<2$ ). The first three factors together explained $38.34 \%$ of the total variance (Table 3 ). If all items suppressed absolute factor loading less than 0.35 , factor I comprised 13 items (2nd, 3rd, 5th, 10th, 11th, 12 th, 13th, 15th, 18th, 19th, 20th, 24th and 28th item), factor II comprised 7 items (7th, 8th, 9th, 17th, 23rd, 30th and 31st item), and factor III comprised four items (21st, 25th, 26th and 27 th item). Factor I could be
Table 2 HCL-32 scores (mean \pm SD) for different levels of current mood state

\begin{tabular}{lllll}
\hline Current mental state & \multicolumn{2}{l}{ BP patients } & \multicolumn{2}{l}{ UP patients } \\
\cline { 2 - 6 } & $\boldsymbol{N}$ & $\begin{array}{l}\text { HCL-32 } \\
\text { score }\end{array}$ & \multicolumn{1}{c}{$\begin{array}{l}\text { HCL-32 } \\
\text { score }\end{array}$} \\
\hline Much worse than usual & 23 & $13.78 \pm 6.20$ & 18 & $10.56 \pm 6.49$ \\
Worse than usual & 35 & $16.41 \pm 5.62$ & 31 & $8.97 \pm 7.60$ \\
A little worse than usual & 38 & $16.78 \pm 4.99$ & 28 & $10.70 \pm 5.03$ \\
Neither better nor worse than & 81 & $15.16 \pm 7.12$ & 46 & $10.35 \pm 6.32$ \\
usual & & & & \\
A little better than usual & 37 & $18.19 \pm 6.11$ & 19 & $14.68 \pm 6.28$ \\
Better than usual & 44 & $16.59 \pm 4.91$ & 5 & $11.00 \pm 2.65$ \\
Much better than usual & 42 & $18.67 \pm 6.45$ & 9 & $15.56 \pm 3.94$ \\
Significance (Kruskal-Wallis test) & - & 0.04 & - & 0.01 \\
\hline
\end{tabular}

described as "active/elated", factor II as "risk-taking" and factor III as "irritable". Other factors for which the eigenvalues were greater than one comprised few items and were difficult to describe for each factor.

\section{Internal consistency}

Internal consistency (Cronbach's alpha) of the Chinese version of the HCL-32 was 0.88 in patients with mood disorders $(N=456)$. Cronbach's alpha of factor I, factor II and factor III were $0.88,0.68$ and 0.74 , respectively.

\section{HCL-32 score comparison between groups}

Mean HCL-32 scores of patients suffering with BP, BP-I or BP-II were statistically higher than those suffering with UP. There was no significant difference in the mean HCL-32 scores of BP-I and BP-II patients (Table 4).

\section{ROC curve analysis}

$R O C$ curve analysis between $B P$ and UP

ROC curve analysis revealed that the HCL-32 could differentiate between BP and UP $(P<0.01)$, and the area under the curve was 0.73 . A screening score of fourteen was the optimal cut-off (sensitivity 0.74 , specificity 0.66 ) between BP and UP. A score of thirteen yielded a sensitivity of 0.77 and a specificity of 0.62 . The sensitivity

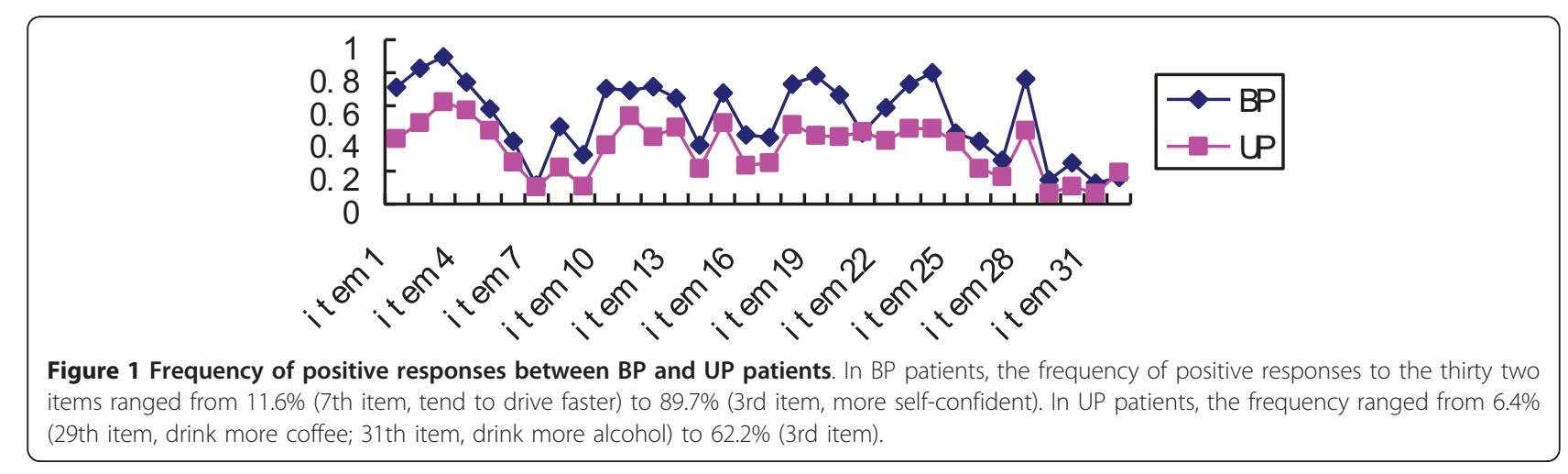


Table 3 Factor loadings of the HCL-32 using factor analysis $(N=456)$

\begin{tabular}{llll}
\hline HCL-32 items & $\begin{array}{l}\text { Active/elated } \\
\text { factor } \\
\text { loadings }\end{array}$ & $\begin{array}{l}\text { Risk-taking } \\
\text { factor } \\
\text { loadings }\end{array}$ & $\begin{array}{l}\text { Irritable } \\
\text { factor } \\
\text { loadings }\end{array}$ \\
\hline 1. need less sleep & 0.32 & 0.17 & 0.2 \\
2. more energetic & $0.65^{*}$ & -0.02 & -0.04 \\
3. more self-confident & $0.61^{*}$ & -0.08 & -0.08 \\
4. enjoy my work more & 0.30 & -0.11 & 0.02 \\
5. more sociable & $0.37^{*}$ & 0.03 & -0.04 \\
6. want to travel more & 0.06 & 0.16 & 0.05 \\
7. drive faster & 0.06 & $0.50^{*}$ & 0.02 \\
8. spend more & 0.17 & $0.63^{*}$ & 0.07 \\
9. take more risks & 0.09 & $0.59^{*}$ & 0.10 \\
10. physically more active & $0.49^{*}$ & 0.08 & -0.09 \\
11. plan more activities & $0.64^{*}$ & -0.04 & 0.02 \\
12. have more ideas/creative & $0.64^{*}$ & 0.28 & -0.04 \\
13. less shy & $0.47^{*}$ & $0.36^{*}$ & -0.02 \\
14. wear more extravagant & 0.27 & 0.29 & 0.13 \\
clothes/make-up & & & \\
15. meet more people & $0.37^{*}$ & 0.10 & 0.09 \\
16. more interested in sex & 0.16 & 0.31 & 0.11 \\
17. more flirtatious & 0.19 & $0.36^{*}$ & 0.10 \\
18. talk more & $0.62^{*}$ & 0.12 & 0.27 \\
19. think faster & $0.79^{*}$ & 0.13 & 0.05 \\
20. make more jokes & $0.54^{*}$ & 0.26 & 0.04 \\
21. more easily distracted & -0.16 & $0.39^{*}$ & $0.53^{*}$ \\
22. engage in more new & 0.24 & 0.31 & -0.06 \\
things & & & \\
23. thoughts jump & $0.36^{*}$ & $0.52^{*}$ & 0.29 \\
24. do more quickly/easily & $0.66^{*}$ & 0.18 & -0.11 \\
25. more impatient/irritable & -0.01 & 0.05 & $0.83^{*}$ \\
26. can be exhausting or & -0.03 & 0.09 & $0.80^{*}$ \\
irritating & & & \\
27. get into more quarrels & 0.07 & 0.24 & $0.64^{*}$ \\
28. mood higher, more & $0.67^{*}$ & 0.16 & -0.07 \\
optimistic & & & \\
29. drink more coffee & 0.03 & 0.12 & 0.08 \\
30. smoke more cigarettes & 0.02 & $0.43^{*}$ & 0.14 \\
31. drink more alcohol & 0.06 & $0.37^{*}$ & 0.12 \\
32. take more drugs & -0.21 & 0.17 & 0.32 \\
Eigenvalue & 18.12 & 2.72 & 2.48 \\
Total variance explained & & & 7.75 \\
\hline
\end{tabular}

*:loading $\geq 0.35$

and specificity at various cut-offs between BP and UP are demonstrated in Figure 2.

\section{ROC curve analysis between BP-I and UP}

ROC curve analysis demonstrated that the HCL-32 could differentiate between BP-I and UP $(P<0.01)$, and the area under the curve was 0.74 . Fourteen was the optimal cut-off between BP-I and UP. The sensitivity and specificity at various cut-offs between BP-I and UP are presented in Figure 3.
Table 4 HCL-32 score comparison between groups

\begin{tabular}{llll}
\hline Groups & Mean HCL-32 score & $\boldsymbol{t}$ & $\boldsymbol{P}$ \\
\hline BP vs. UP & $16.51 \pm 6.22$ vs. $10.90 \pm 6.43$ & 9.05 & $P<0.01$ \\
BP-I vs. UP & $16.91 \pm 6.35$ vs. $10.90 \pm 6.43$ & 8.98 & $P<0.01$ \\
BP-I vs. BP-II & $16.91 \pm 6.35$ vs. $15.15 \pm 5.92$ & 1.88 & $P>0.05$ \\
BP-II vs. UP & $15.15 \pm 5.92$ vs. $10.90 \pm 6.43$ & 4.82 & $P<0.01$ \\
\hline
\end{tabular}

\section{ROC curve analysis between BP-I and BP-II}

The HCL-32 could not distinguish between BP-I and BP-II $(P=0.08)$ using ROC curve analysis. The area under the curve was 0.57 .

ROC curve analysis between BP-II and UP

ROC curve analysis revealed that the HCL-32 could discriminate between BP-II and UP $(P<0.01)$, and the area under the curve was 0.69 . Thirteen was the optimal cut-off to discriminate between BP-II and UP. The sensitivity and specificity at various cut-offs between BP-II and UP are presented in Figure 4.

\section{Positive Predictive Value (PPV) and Negative Predictive Value (NPV)}

At a cut-off of thirteen between BP and UP, the PPV was $77 \%$ and the NPV was $56 \%$. At a cut-off of fourteen between BP and UP, the PPV was $78 \%$ and NPV was $54 \%$.

\section{Discussion}

Bipolar disorder is very common and the lifetime prevalence of bipolar disorder spectrum is approximately $4.5 \%$ in the general population [16,17]. Moreover, bipolar disorder is associated with substantial impairments in productive and social roles $[18,19]$. The HCL-32 is a convenient instrument for screening bipolar disorders, and psychiatrists in several countries use it in practice $[12-15,20,21]$. China is the most populated country in the world. Therefore, a study concerning the use of the HCL-32 in China is important.

The mean age of BP patients was significantly lower than that of UP patients in this study, and this is comparable with samples used for similar studies $[12,14,20]$. The percentage of female UP patients was higher than the percentage of female BP patients. This could reflect the fact that rates of major depression are higher in females than in males, and they are comparable for bipolar disorder [22]. Differences concerning the mean age and sex ratio between BP and UP patients could have resulted from enrolling individuals consecutively. There were more BP-I patients than BP-II patients as inpatients as well as outpatients were enrolled in the study (more inpatients suffer from BP-I than BP-II).

The mean HCL-32 scores were statistically different between groups, defined according to their current mental state in BP and UP. Therefore, there was a possible 

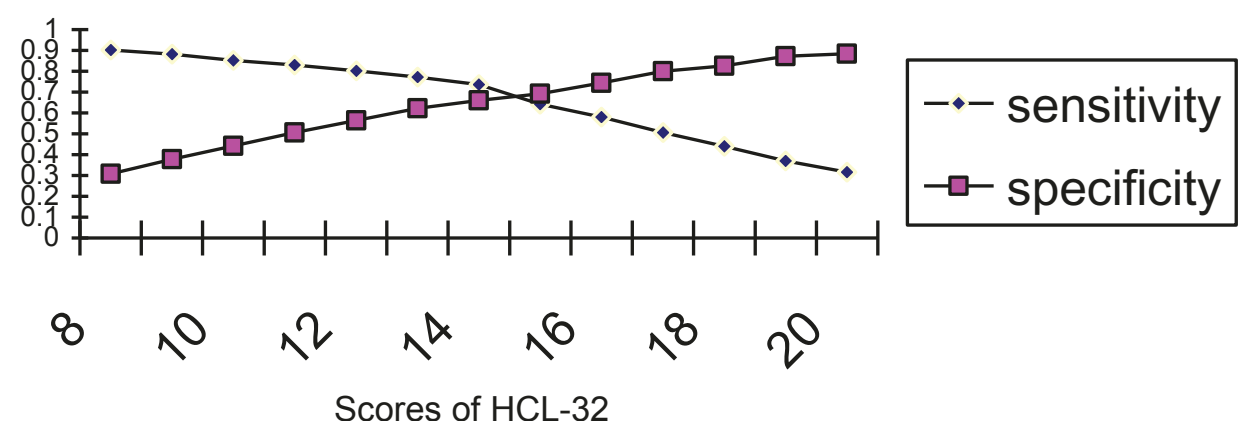

Figure 2 Sensitivity and specificity at various cut-offs between BP and UP.

impact of current mental state on HCL-32 scores of patients with mood disorders. This result is similar to that of a Taiwanese study [14], but different from results obtained in Europe [12,15]. Low correlation coefficients were evident between current mental state and the HCL-32 score in BP $(r=0.13)$ and UP $(r=0.23)$ patients. The impact of current mental state on the HCL-32 score is likely to be low and limited.

A three-factor solution using factor analysis in this study is different from the results obtained in the European and Taiwanese studies [12,14]. Angst reported two factors ("active/elated" and "risk-taking/irritable") from the study carried out in Europe [12]. Item 9 (take more risks) is included in factor II in the European study, but not in factor I or factor II in the Taiwanese study [14]. Combining the factor II and factor III items in the present study is similar to those of factor II in the European study. The items of factor II in the Taiwanese study are similar to those of factor III in this study [14].

Cronbach's alpha for the HCL-32 was 0.88 in the present study. This is comparable to the results from other studies (0.82 in Italian sample, 0.86 in Swedish sample, 0.90 in Spanish sample and 0.88 in Taiwanese sample)
[12-15]. The internal consistency of the HCL-32 was good for various ethnic samples.

The frequency of positive responses to four items (7th, drive faster; $21 \mathrm{st}$, more easily distracted; $25 \mathrm{th}$, more impatient/irritable; 32nd, take more drugs) in BP patients was not significantly higher than for UP sufferers. The percentage of people who own a car in China is low, and this could explain why the frequency of the 7th item (drive faster) was low in BP (11.6\%) and UP (10.3\%) patients. The reason for no significant difference for the three other items is unclear.

The HCL-32 could distinguish between BP and UP, BP-I and UP, BP-II and UP, but not between BP-I and BP-II in the present study. These results are comparable to those of the European study [12]. However, HCL-32 can distinguish between BP-I and BP-II, with the optimal cut-off of 21, in the Taiwan study [14]. Subjects in the present study and that carried out in Taiwan were Chinese. In the European and Taiwanese studies, the duration criterion for hypomania was two days but in the present study it was a minimum of four days. The ratio of BP-I and BP-II patients between the Taiwanese study and the European study are similar (66/94 vs. 105/164).
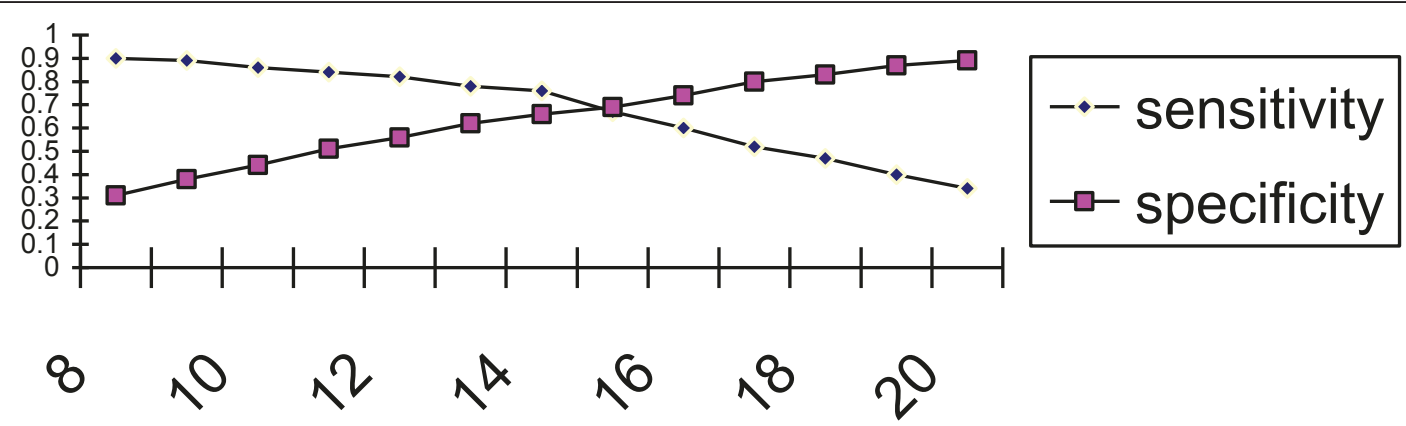

Scores of HCL-32

Figure 3 Sensitivity and specificity at various cut-offs between BP-I and UP. 


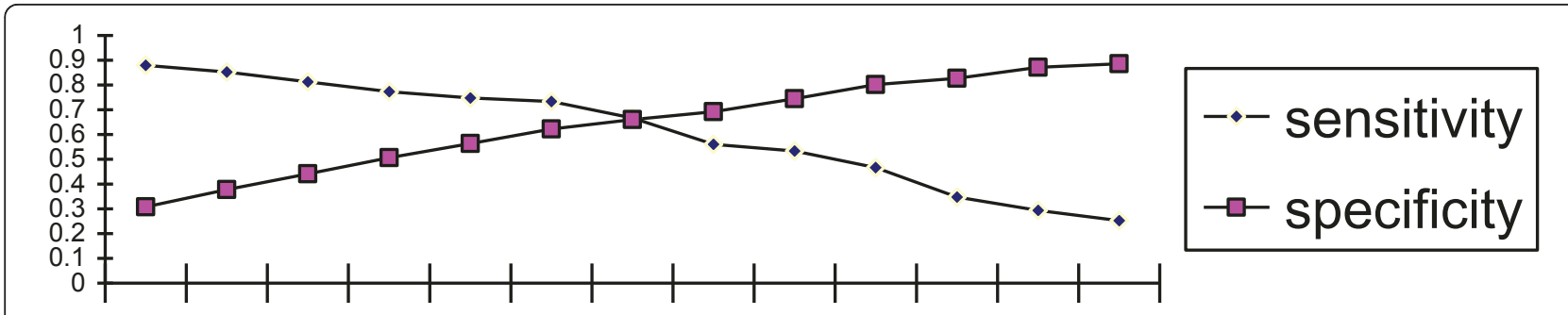

\section{0}

\section{Scores of HCL-32}

Figure 4 Sensitivity and specificity at various cut-offs between BP-II and UP. A cut-off of thirteen had sensitivity of 0.73 and a specificity of 0.62 between BP-II and UP. A cut-off of fourteen had a sensitivity 0.67 and a specificity 0.66 between BP-II and UP.

In this study, fourteen was chosen as the optimal cut-off between BP and UP if BP was not divided into BP-I and BP-II. This was similar to the results from other studies $[12,14]$. In this study, the HCL-32 could discriminate between BP-I and UP, with the best cut-off being fourteen. In a UK study, the HCL-32 could distinguish between BP-I and UP, with the best cut-off being twenty [21].

The HCL-32 could discriminate between BP-II and UP, with the optimal cut-off of thirteen. The difficulty in distinguishing between BP and UP is related to difficulties in discriminating between BP-II and UP in psychiatric settings. Patients with BP-I are less likely to be misdiagnosed than those with BP-II. The results from the current study suggest that the optimal cut-off between BP-II and UP should be used, particularly when considering the continuum of mood disorders. BP-II is closer to UP than BP-I [23]. The sensitivity of detecting BP-II could be improved if thirteen is used as the optimal cut-off between BP and UP. There were more BP-II patients than BP-I patients $[16,17,24-26]$. High sensitivity is important for a screening instrument (cut-off thirteen, sensitivity 0.77 , specificity 0.62 ; cut-off fourteen, sensitivity 0.74 , specificity 0.66 ). From a clinical perspective, a screening questionnaire must have good sensitivity even if that increases false positives because of lower specificity [27].

The PPV at a cut-off of thirteen was $1 \%$ lower than that at a cut-off of fourteen, while the NPV was higher than $2 \%$. The PPV and NPV at the cut-off of thirteen were better than at a cut-off of 14 but the advantage was not great.

There were limitations in the present study. The number of BP-I patients was greater than the number of BPII patients, and there were differences in terms of the mean age and sex ratio between BP and UP patients. The duration of the mood disorders were not evaluated in the current study as diagnoses were correlated to the duration of mood disorders.

\section{Conclusions}

The psychometric properties of the simplified Chinese version of the HCL-32 were demonstrated to be satisfactory using a clinical sample in China. The best cut-off between BP-II and UP should be regarded as the optimal cut-off between BP and UP when using the HCL32. Furthermore, 13 can be used as the optimal screening cut-off between BP and UP in psychiatric settings in China.

\section{Acknowledgements and Funding}

We thank Dr. Alex Gamma (Zurich University Psychiatric Hospital,

Switzerland) for his suggestions concerning the manuscript. This study was supported by a grant from the National Natural Science Foundation of China (30830046 to Ling-jiang Li), the National Science and Technology Program of China (2007BAl17B02 to Ling-jiang Li), the National 973 Program of China (2009CB918303 to Ling-jiang Li), Program of Chinese Ministry of Education (20090162110011 to Ling-jiang Li) and grant (200602032 to Haichen Yang) from the scientific and technological bureau of Shenzhen city.

\section{Author details}

${ }^{1}$ Mental Health Institute, the 2nd Xiangya Hospital, Central South University, No. 139 Renmin Zhong Road, Changsha 410011, China. ${ }^{2}$ Division of Mood Disorders, Shenzhen Mental Health Centre, Shenzhen 518020, China. ${ }^{3}$ Division of Mood Disorders, Shanghai Mental Health Centre, Shanghai Jiaotong University School of Medicine, Shanghai 200030, China. ${ }^{4}$ Zurich University Psychiatric Hospital, Switzerland.

\section{Authors' contributions}

Authors $L L$ and HY designed the study and developed the protocols. $L L$ is the tutor of HY. Authors LL, HY, TL and CY carried out literature searches and analyses. Authors $L L, H Y, T L, H P, C L$ and $R H$ undertook the statistical analysis and prepared the first draft of the manuscript. All authors were interviewers. Authors HY and TL oversaw the research in Shenzhen. Authors $C Y$ and YF directed the research in Shanghai. All authors read and approved the final manuscript.

\section{Authors' information}

${ }^{1}$ Mental Health Institute, the 2nd Xiangya Hospital, Central South University, No. 139 Renmin Zhong Road, Changsha 410011, PR China. ${ }^{2}$ Division of 
Mood Disorders, Shenzhen Mental health centre, Shenzhen 518020, PR China. ${ }^{3}$ Division of Mood Disorders, Shanghai Mental Health Centre, Shanghai Jiaotong University School of Medicine, Shanghai 200030, PR China. ${ }^{4}$ Zurich University Psychiatric Hospital, Switzerland.

\section{Competing interests}

The authors declare that they have no competing interests.

Received: 21 November 2010 Accepted: 15 May 2011

Published: 15 May 2011

\section{References}

1. Salvi V, Fagiolini A, Swartz HA, Maina G, Frank E: The use of antidepressants in bipolar disorder. J Clin Psychiatry 2008, 69:1307-1318.

2. Bowden $\mathrm{CL}: \mathrm{A}$ different depression: clinical distinctions between bipolar and unipolar depression. J Affect Disord 2005, 84:117-125.

3. Stensland MD, Schultz JF, Frytak JR: Depression diagnoses following the identification of bipolar disorder: costly incongruent diagnoses. BMC Psychiatry 2010, 10:39.

4. Ghaemi SN, Sachs GS, Chiou AM, Pandurangi AK, Goodwin K: Is bipolar disorder still underdiagnosed? Are antidepressants overutilized? J Affect Disord 1999, 52:135-144.

5. Hirschfeld RM, Lewis L, Vornik LA: Perception and impact of bipolar disorder: how far have we really come. Results of the national depressive and manic-depressive association 2000 survey of individual with bipolar disorder. J Clin Psychiatry 2003, 64:161-174.

6. Zhang HX, XU JM, Ji JL: Diagnosis and treatment for outpatients with bipolar affective disorder. Chin J Behavioral Medical Science 2002, 11:270-171.

7. Judd LL, Akiskal HS: Depressive episodes and symptoms dominate the longitudinal course of bipolar disorder. Curr Psychiatry Rep 2003, 5:417-418.

8. Hantouche EG, Akiskal HS, Lancrenon S, Allilaire JF, Sechter D, Azorin JM, Bourgeois M, Fraud JP, Chatenet-Duchene L: Systematic clinical methodology for validating bipolar-II disorder: data in mid-stream from a French national multi-site study (EPIDEP). J Affect Disord 1998, 50:163-173.

9. American Psychiatric Association: Practice guideline for the treatment of patients with bipolar disorder(revision). Am J Psychiatry 2002, 159 (4 suppl):1-50

10. Suppes $T$, Leverich GS, Keck PE, Nolen WA, Denicoff KD, Altshuler $L L$, McElroy SL, Rush AJ, Kupka R, Frye MA, Bickel M, Post RM: The Stanley foundation Bipolar Treatment outcome Network .Il. Demographics and illness characteristic of the first 261 patients. J Affect Disord 2001, 67:45-49.

11. Dunner DL, Tay LK: Diagnostic reliability of the history of hypomania in bipolar II patients and patients with major depression. Comprehensive Psychiatry 1993, 34:303-307.

12. Angst J, Adolfsson R, Bennazzi F, et al: The HCL-32: Towards a selfassessment tool for hypomanic symptoms in outpatients. $J$ Affect Disord 2005, 88:217-233.

13. Carta GM, Hardoy MC, Cadeddu M, Murru A, Campus A, Morosini PL, Gamma A, Angst J: The accuracy of the Italian version of the Hypomania Checklist (HCL-32) for the screening of bipolar disorders and comparison with the Mood Disorder Questionnaire (MDQ) in a Clinical sample. Clin Pract Epidemo Ment Health 2006, 2:1-5.

14. Wu YS, Angst J, Ou CS, Chen HC, Lu RB: Validation of the Chinese version of the Hypomania Checklist(HCL-32) as an instrument for detecting hypo(mania) in patients with mood disorders. J Affect Disord 2008, 106:133-143.

15. Vieta E, Sanchez-Moreno J, Bulbena A, Chamorro L, Ramos JL, Artal J, Perez F, Oliveras MA, Valle J, Lahuerta J, Angst J: Cross validation with the mood disorder questionnaire (MDQ) of an instrument for the detection of hypomania in Spanish: The 32-item hypomania symptom checklist (HCL-32). J Affect Disord 2007, 101:43-55.

16. Kessler RC, Berglund P, Demler O, Jin R, Merikangas KR, Walters EE: Lifetime prevalence and age-of-onset distributions of DSM-IV disorders in the national comorbidity survey replication. Arch Gen Psychiatry 2005, 62:593-602.
17. Kessler RC, Akiskal HS, Angst J, Guyer M, Hirschfeld RM, Merikangas KR Stang PE: Validity of the assessment of bipolar spectrum disorders in the WHO CIDI 3.0. J Affect Disord 2006, 69:259-269.

18. Das Gupta R, Guest JF: Annual cost of bipolar disorder to UK society. The British Journal of Psychiatry 2002, 180:227-233.

19. Kleinman L, Lowin A, Flood E, Gandhi G, Edgell E, Revicki D: Costs of bipolar disorder. Pharmacoeconomics 2003, 21:601-622

20. Kim B, Wang RH, Son Jl, Kim CY, Joo YH: Bipolarity in depressive patients without histories of diagnosis of bipolar disorder and the use of the mood disorder questionnaire for the detecting bipolarity. Comprehensive Psychiatry 2008, 49:469-475.

21. Forty L, Smith D, Jones J, Jones I, Caesar S, Fraser C, Gordon-Smith K, Craddock N: Identifying hypomanic features in major depressive disorder using the hypomania checklist (HCL-32). J Affect Disord 2009, 114:68-73.

22. Weissman MM, Bland R, Joyce PR, Newman S, Wells JE, Wittchen HU: Sex difference in rates of depression: cross-national perspectives. J Affect Disord 1993, 29:77-84.

23. Akiskala HS, Benazzi F: Atypical depression: a variant of bipolar II or a bridge between unipolar and bipolar II? J Affect Disord 2005, 84:209-217.

24. Szadocczky E, Papp Z, Vitrai J, Rihmer Z, Furedi J: The prevalence of major depressive and bipolar disorder in Hungary: results from a national epidemiologic survey. J Affect Disord 1998, 50:153-162.

25. Faravelli C, Rosi S, Alessandra SM, Lampronti L, Amedei SG, Rana N: Threshold and subthreshold bipolar disorders in the Sesto Fiorentino study. J Affect Disord 2006, 94:111-119.

26. Judd LL, Akiskal HS: The prevalence and disability of bipolar spectrum disorders in the US population: re-analysis of the ECA database taking into account subthreshold cases. J Affect Disord 2003, 73:123-131.

27. Zimmerman M, Postermak MA, Chelminski I, Solomon DA: Using questionnaire to screen for psychiatric disorders: a comment on a study of screening for bipolar disorder in the community. J Clin Psychiatry 2004, 65:605-610.

\section{Pre-publication history}

The pre-publication history for this paper can be accessed here: http://www.biomedcentral.com/1471-244X/11/84/prepub

\section{doi:10.1186/1471-244X-11-84}

Cite this article as: Yang et al:. Validity of the 32-item Hypomania Checklist (HCL-32) in a clinical sample with mood disorders in China. BMC Psychiatry 2011 11:84.

\section{Submit your next manuscript to BioMed Central and take full advantage of:}

- Convenient online submission

- Thorough peer review

- No space constraints or color figure charges

- Immediate publication on acceptance

- Inclusion in PubMed, CAS, Scopus and Google Scholar

- Research which is freely available for redistribution

Submit your manuscript at www.biomedcentral.com/submit
C) Bïomed Central 